\title{
Reduced fertility caused by meiotic defects and micronuclei formation during microsporogenesis in xBrassicoraphanus
}

\author{
Hosub Shin ${ }^{1}$. Hye Rang Park ${ }^{1}$ Jeong Eun Park ${ }^{1}$. Seung Hwa Yu ${ }^{2}$ Gibum Yi ${ }^{3}$. Jung Hyo Kim ${ }^{2}$. Wonjun Koh ${ }^{1}$. \\ Hyun Hee Kim ${ }^{4} \cdot$ Soo-Seong Lee ${ }^{5} \cdot$ Jin Hoe Huh ${ }^{1,2,3,6}$ (1)
}

Received: 10 January 2021 / Accepted: 13 January 2021 / Published online: 8 February 2021

(c) The Author(s) 2021

\begin{abstract}
Background Hybridization and polyploidization events are important driving forces in plant evolution. Allopolyploids formed between different species can be naturally or artificially created but often suffer from genetic instability and infertility in successive generations. xBrassicoraphanus is an intergeneric allopolyploid obtained from a cross between Brassica rapa and Raphanus sativus, providing a useful resource for genetic and genomic study in hybrid species.

Objective The current study aims to understand the cause of hybrid sterility and pollen abnormality in different lines of synthetic $\mathrm{x}$ Brassicoraphanus from the cytogenetic perspective.

Methods Alexander staining was used to assess the pollen viability. Cytogenetic analysis was employed to monitor meiotic chromosome behaviors in pollen mother cells (PMCs). Origins of parental chromosomes in xBrassicoraphanus meiocytes were determined by genome in situ hybridization analysis.

Results The $\mathrm{xBrassicoraphanus} \mathrm{lines} \mathrm{BB \# 4} \mathrm{and} \mathrm{BB \# 6} \mathrm{showed} \mathrm{high} \mathrm{rates} \mathrm{of} \mathrm{seed} \mathrm{abortion} \mathrm{and} \mathrm{pollen} \mathrm{deformation.} \mathrm{Abnormal}$ chromosome behaviors were observed in their PMCs, frequently forming univalents and inter-chromosomal bridges during meiosis. A positive correlation also exists between meiotic defects and the formation of micronuclei, which is conceivably responsible for unbalanced gamete production and pollen sterility.

Conclusion These results suggest that unequal segregation of meiotic chromosomes, due in part to non-homologous interactions, is responsible for micronuclei and unbalanced gamete formation, eventually leading to pollen degeneration and inferior fertility in unstable xBrassicoraphanus lines.
\end{abstract}

Keywords Intergeneric hybrids $\cdot$ Pollen fertility $\cdot$ Meiosis $\cdot$ Micronucleus $\cdot x$ Brassicoraphanus

Hosub Shin, Hye Rang Park and Jeong Eun Park authors contributed equally.

Jin Hoe Huh

huhjh@snu.ac.kr

1 Department of Agriculture, Forestry and Bioresources, Seoul National University, Seoul 08826, South Korea

2 Interdisciplinary Program in Agricultural Genomics, Seoul National University, Seoul 08826, South Korea

3 Plant Genomics and Breeding Institute, Seoul National University, Seoul 08826, South Korea

4 Department of Life Science, Chromosome Research Institute, Sahmyook University, Seoul 01795, South Korea

5 BioBreeding Institute, Anseong 17544, South Korea

6 Research Institute of Agriculture and Life Sciences, Seoul National University, Seoul 08826, South Korea

\section{Introduction}

Hybridization and polyploidization are driving forces in plant evolution, providing phenotypic variability and competitiveness for adaptation (Chen 2007; Cheng et al. 2018; Soltis and Soltis 2009; Van de Peer et al. 2017; Wendel 2000). Polyploidy is predominantly observed in flowering plants (Masterson 1994), and many domesticated crop species, including oilseed rape (Chalhoub et al. 2014), mustard (Yang et al. 2016), wheat (International Wheat Genome Sequencing 2014), cotton (Zhang et al. 2015), and strawberry (Edger et al. 2019), have undergone polyploidization events. Although there are many spontaneous hybrids in nature, successful speciation through hybridization is not straightforward, owing to hybridization barriers that are frequently observed in early generations of hybrid offspring, manifested as outbreeding depression, reduced viability, and 
reduced fertility (Abbott et al. 2013; Todesco et al. 2016). Several mechanisms have been proposed to explain hybridization barriers such as abnormal endosperm development, self-incompatibility and meiotic failure during gametogenesis (Dion-Cote and Barbash 2017).

Meiosis is the process by which four haploid gametes are generated from a diploid progenitor cell for sexual reproduction. During this process, the progenitor cell is divided into the tetrad through two successive meiotic cell divisions, which entails strand exchanges between homologous chromosomes and precise segregation of sister chromatids, and for the production of male gametophytes, four microspores are released from the tetrad and develop into pollen grains. Therefore, successful meiosis during gamete formation is an essential step to produce fertile pollen in plant reproduction. In particular, abnormal interactions between non-homologous chromosomes may have detrimental consequences, including reduced pollen viability in allopolyploids. Many studies have reported non-homologous chromosome pairing in artificially synthesized allopolyploid plants such as rapeseed, tobacco and wheat (Chen et al. 2018; Xiong et al. 2011; Zhang et al. 2013). Unpaired univalents, homoeologous bivalents, or multivalents induced by aberrant chromosome pairings are likely to cause meiotic chromosomes to mis-segregate, resulting in impaired fertility (Cifuentes et al. 2010; Szadkowski et al. 2010).

The Brassicaceae family is well known for species diversification through allopolyploidization events between related species as described by the "U's triangle" (U 1935), wherein three Brassica species B. napus (AACC; $2 \mathrm{n}=4 \mathrm{x}=38), B$. juncea $(\mathrm{AABB} ; 2 \mathrm{n}=4 \mathrm{x}=36)$ and $B$. carinata $(\mathrm{BBCC} ; 2 \mathrm{n}=4 \mathrm{x}=34)$ emerged naturally by hybridization and polyploidization among three diploid species $B$. rapa $(\mathrm{AA} ; 2 \mathrm{n}=2 \mathrm{x}=20), B$. nigra $(\mathrm{BB} ; 2 \mathrm{n}=2 \mathrm{x}=16)$ and $B$. oleracea $(C C ; 2 n=2 x=18)$. Besides interspecific hybridization, an intergeneric hybridization event may arise sporadically between two divergent species that belong to different genera. An exemplary case of such hybridizations is $\mathrm{xBrassi-}$ coraphanus, an intergeneric allotetraploid synthesized from a cross between B. rapa and Raphanus sativus. The $\mathrm{xBras}$ sicoraphanus cultivar BB\#1 was reported to display exceptional phenotypic uniformity and high fertility (Lee et al. 2002, 2011, 2017), and unlike many other synthetic hybrids, showed normal diploid-like meiotic behaviors (Park et al. 2020). There are several lines of $x$ Brassicoraphanus besides BB\#1. The four $\mathrm{xBrassicoraphanus} \mathrm{cultivars} \mathrm{BB \# 4,} \mathrm{BB \# 6,}$ $\mathrm{BB} \# 12$ and BB\#50 were generated from a cross between $B$. rapa $\mathrm{cv}$. Jeonseung $(2 \mathrm{n}=2 \mathrm{x}=20)$ and $R$. sativus $\mathrm{cv}$. Taebaek $(2 \mathrm{n}=2 \mathrm{x}=18)$ (Lee et al. 2002). The first generation

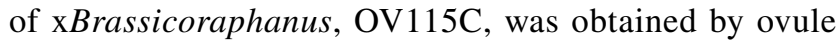
culture and colchicine treatment (Fig. 1a) (Lee et al. 2002). From OV115C, lines BB\#4 and BB\#6 were generated by microspore culture, and $\mathrm{BB} \# 12$ and $\mathrm{BB} \# 50$ were developed by microspore culture with the treatment of $N$-methyl- $N$-nitroso-urethane (NMU).

Notably, lines BB\#4 and BB\#6 have severe pollen abnormality and seed abortion, which is reminiscent of a post-zygotic hybridization barrier often observed in many hybrid offspring. In this study, we compared seed yield, pollen shape and viability, tetrad formation, and meiotic chromosome behaviors in pollen mother cells (PMCs) among different $\mathrm{x}$ Brassicoraphanus lines. We frequently detected a

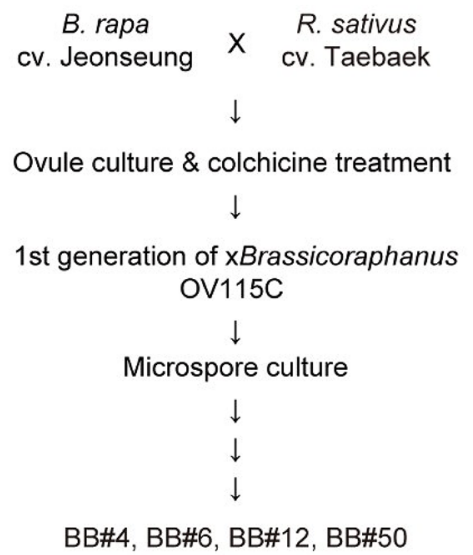

b

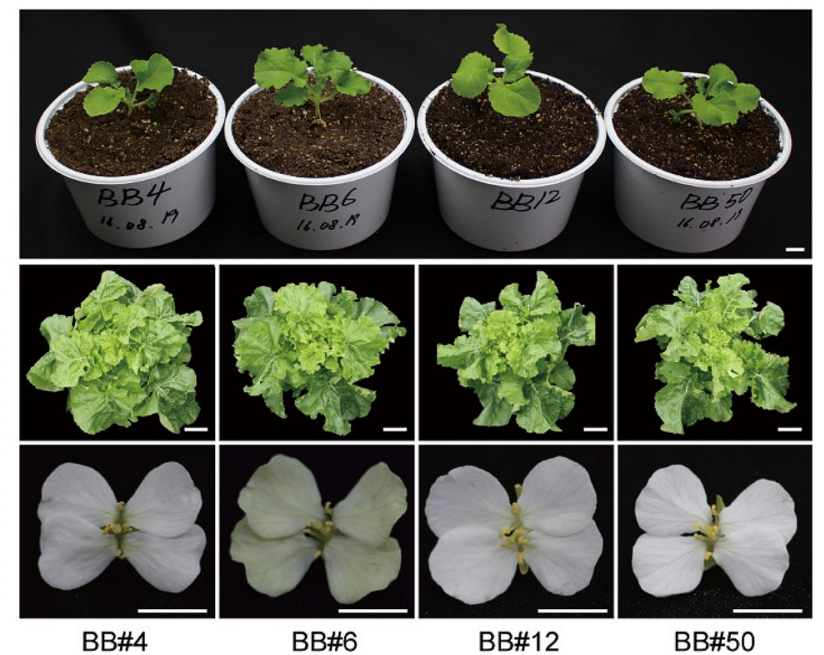

Fig. 1 Pedigree and morphology of four $\mathrm{xBrassicoraphanus} \mathrm{lines.} \mathrm{a}$ The first generation of xBrassicoraphanus, OV115C, was obtained by a cross between $B$. rapa cv. Jeonseung and $R$. sativus $\mathrm{cv}$. Taebaek. Four $\mathrm{xBrassicoraphanus} \mathrm{lines} \mathrm{were} \mathrm{developed} \mathrm{from} \mathrm{OV115C} \mathrm{through}$ microspore culture. b Morphology of seedlings (upper), mature plants (middle) and flowers (lower). Scale bar $=1 \mathrm{~cm}$ (flower and seedling) or $10 \mathrm{~cm}$ (mature plant) 
micronucleus formation at the tetrad stage in $\mathrm{x}$ Brassicoraphanus lines BB\#4 and BB\#6, from which more abnormal pollen was generated. In addition, univalent chromosomes and chromosome bridges were observed during microsporogenesis, presumably leading to micronucleus formation in the tetrad and pollen abnormality. Our data strongly suggest that unequal segregation of meiotic chromosomes during microsporogenesis is likely responsible for the formation of micronuclei and unbalanced gametes in a pollen tetrad, eventually leading to pollen deformation and reduced fertility in unstable xBrassicoraphanus lines.

\section{Materials and methods}

\section{Plant materials and growth conditions}

Seeds of xBrassicoraphanus cultivar BB\#4, BB\#6, BB\#12 and $\mathrm{BB} \# 50$ were sterilized in 50\% hypochlorite solution with $0.1 \%$ Triton X-100 for $90 \mathrm{~s}$, followed by 10 washes with sterile distilled water. The seeds were plated on Murashige and Skoog medium (Duchefa, Netherlands) supplemented with 2\% (w/v) sucrose and $0.8 \%(\mathrm{w} / \mathrm{v})$ plant agar (Murashige and Skoog 1962). Plates were incubated at $24{ }^{\circ} \mathrm{C}$, with a daily cycle of $16 \mathrm{~h}$ of light and $8 \mathrm{~h}$ of dark, for 2 weeks. Plants were vernalized at $4{ }^{\circ} \mathrm{C}$, with the same light cycle, for 4 weeks. Plants were transferred to soil in pots and placed in the glasshouse with the same light conditions.

\section{Alexander staining}

Alexander's stain solution was prepared and used to distinguish between viable and nonviable pollen (Alexander 1969). Mature floral buds were collected and fixed in Carnoy's solution (alcohol:acetic acid, 3:1, v/v), then placed on microscope slides and dissected to release the pollen. Stain solution (40-60 $\mu \mathrm{l}$ ) was applied before the sample dried completely. After staining the pollen grains, a coverslip was placed over the sample and uniform pressure was applied. The slides were examined using an Axioskop2 microscope equipped with an Axiocam 506 color CCD camera (Zeiss, Germany).

\section{Scanning electron microscopy (SEM)}

For SEM evaluation, properly dried pollen of each cultivar was dusted onto aluminum stubs with a thermosensitive glue. The sample on each stub was sputter-coated with a $30 \mathrm{~mA}$ - thick layer of platinum for $200 \mathrm{~s}$ at $20 \mathrm{~mA}$, using a Sputter Coater (Leica, Austria). Pollen grains were observed using a field emission scanning electron microscope (Zeiss, Germany).

\section{Genome in situ hybridization (GISH)}

Floral buds were fixed in the aceto-ethanol $(1: 3 \mathrm{v} / \mathrm{v})$ solution for $24 \mathrm{~h}$ and stored at $-20{ }^{\circ} \mathrm{C}$ in $70 \%$ ethanol until use. The fixed buds were rinsed in distilled water. Slide preparation, probe preparation and hybridization were performed according to published protocols (Park et al. 2020). Slides were imaged using an Axioskop2 microscope equipped with an Axiocam 506 color CCD camera (Zeiss, Germany).

\section{Results}

\section{Variability of seed formation among xBrassicoraphanus lines}

Four $\mathrm{xBrassicoraphanus} \mathrm{lines} \mathrm{BB \# 4,} \mathrm{BB \# 6,} \mathrm{BB \# 12} \mathrm{and}$ $\mathrm{BB} \# 50$ were previously generated from a cross between commercial cultivars of $B$. rapa and $R$. sativus followed by microspore culture and colchicine treatment (Fig. 1a; Lee et al. 2002, 2011). All four xBrassicoraphanus lines showed very similar phenotypes from young seedling to adult stage (Fig. 1b). However, flower color was pale yellow in BB\#6, while flowers of the other three lines were white (Fig. 1b). To assess variability in seed formation rate, xBrassicoraphanus lines were self-pollinated in the glasshouse, and normal and aborted seeds in siliques were counted. Average numbers of total seeds per silique were $3.38 \pm 0.21$ in $\mathrm{BB} \# 4,2.20 \pm 0.26$ in $\mathrm{BB} \# 6,1.97 \pm 0.18$ in $\mathrm{BB} \# 12$ and $2.32 \pm 0.21$ in BB\#50 (Fig. 2a). Proportions of normal seeds per silique were $52.23 \pm 2.42 \%$ in $\mathrm{BB} \# 4,52.51 \pm 6.17 \%$ in $\mathrm{BB} \# 6,72.20 \pm 1.56 \%$ in BB\#12 and $81.25 \pm 3.34 \%$ in BB\#50 (Fig. 2b). Although production of normally developed seeds did not differ significantly among xBrassicoraphanus lines (ANOVA, $p=0.418$ ), the aborted seed ratios were significantly lower in BB\#12 and BB\#50 than in BB\#4 and BB\#6 (ANOVA with Duncan's multiple test, $p=4.53 \times 10^{-04}$ ). These data suggest that each $\mathrm{xBrassicoraphanus} \mathrm{line} \mathrm{has}$ similar phenotypes in most traits but substantially differs for seed production efficiency.

\section{Differential pollen viability in xBrassicoraphanus}

Pollination is the first step in sexual reproduction, and thus, fertile pollen grains are required for fertilization and seed development. To investigate the fertility of male gametophytes in BB\#4, BB\#6, BB\#12 and BB\#50, pollen shapes were observed by SEM (Fig. 3a), and the viability was determined by staining pollen with Alexander's stain solution (Fig. 3b). All normal pollen grains of B. rapa and R. sativus have a prolate spheroid or tricolpate shape, in accordance with previous studies (Goda 2018; Hossain et al. 1990), but two different types of pollen grains were also observed in 
a

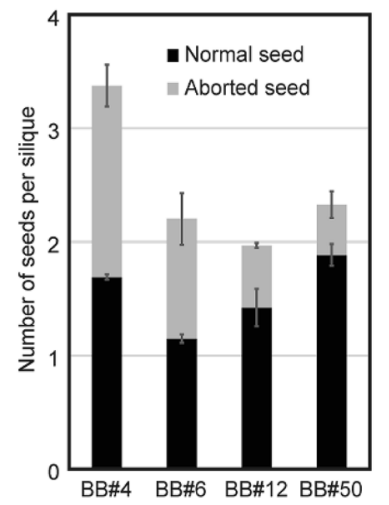

b

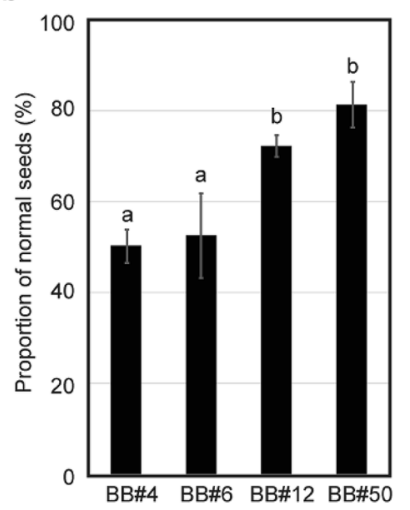

Fig. 2 Seed counts per silique and proportions of normal seeds in four xBrassicoraphanus lines. a Seed numbers per silique. Black and grey bars represent the numbers of normal and aborted seeds, respectively. b Proportion of normal seeds per silique. More than one hundred siliques from two independent plants were analyzed for each line. Error bars indicate standard deviations and differences among $\mathrm{x}$ Brassicoraphanus lines were tested with one-way ANOVA, as indicated with letters, using Duncan's multiple test $(p<0.05)$

all xBrassicoraphanus lines. One pollen type had a spheroid shape phenotypically similar to the parental species, while the other displayed a spherical shape and the grains were noticeably smaller (Fig. 3a). When stained for viability with Alexander's solution, large spheroid pollen grains were all stained in red, whereas the deformed and shrunken pollen grains of $\mathrm{xBrassicoraphanus} \mathrm{lines} \mathrm{remained} \mathrm{unstained,}$ indicative of pollen sterility. By counting the number of normal and abnormal pollen grains in the xBrassicoraphanus lines, we determined the proportion of normally shaped pollen to be $22.38 \pm 8.61 \%$ in BB\#4, $28.50 \pm 8.50 \%$ in BB\#6, $31.04 \pm 7.59 \%$ in BB\#12 and $44.39 \pm 3.70 \%$ in BB\#50 (Fig. 3c), revealing approximately a twofold greater viability in BB\#50 than BB\#4 (ANOVA with Duncan's multiple test, $p=8.95 \times 10^{-4}$ ). This observation suggests that superior pollen viability observed in $\mathrm{BB} \# 50$ might be associated with a higher normal seed development rate for reproductive success.

\section{Formation of micronuclei in xBrassicoraphanus lines BB\#4 and BB\#6}

At the end of microsporogenesis, microspores are released from the tetrad and develop into mature pollen. Thus, proper tetrad formation should precede microgametogenesis to produce functional pollen. To investigate the formation of tetrads in $\mathrm{x}$ Brassicoraphanus PMCs, male gametophytes from buds $1-2 \mathrm{~mm}$ in size were stained with Alexander's staining solution. Most tetrads in xBrassicoraphanus lines consisted of four individual microspores, but additional micronuclei were frequently observed in a few cases (Fig. 4a). The

frequencies of tetrads containing micronuclei were $6.98 \%$ in $\mathrm{BB} \# 4,4.79 \%$ in $\mathrm{BB} \# 6,2.28 \%$ in $\mathrm{BB} \# 12$ and $0.74 \%$ in $\mathrm{BB} \# 50$ (Table 1). It is presumed that microspores released from unbalanced tetrads are unable to develop into normal pollen grains due to an incomplete chromosome complement, and production of abnormal microspores is likely to cause a reduction in pollen fertility observed in $\mathrm{xBrassic}$ oraphanus lines BB\#4 and BB\#6.

Indeed, a positive relationship was revealed between micronucleus formation and pollen abnormality in these synthetic $\mathrm{x}$ Brassicoraphanus lines (Pearson's correlation coefficient, $r=0.9222, p=0.0389$, one-tailed test), suggesting that increased pollen viability observed in BB\#50 might result from less micronuclei formation during microsporogenesis.

\section{Abnormal meiotic chromosome behaviors during microsporogenesis in xBrassicoraphanus PMCs}

In many newly synthesized interspecific hybrids, abnormal meiotic chromosome behaviors were associated with nonhomologous chromosome interactions, resulting in unbalanced gamete formation and reduced fertility (MartinezPerez and Colaiacovo 2009). To investigate the cause of micronucleus formation observed in xBrassicoraphanus lines, we compared meiotic chromosomal behaviors in PMCs of BB\#50 and BB\#4 (Fig. 5). At leptotene, the first substage of prophase I, chromosome condensation was initiated (Fig. 5a, i), and pairing of homologous chromosomes occurred at zygotene (Fig. 5b, j). At pachytene, homologous chromosomes were synapsed (Fig. $5 \mathrm{c}, \mathrm{k}$ ), and a total of 19 bivalents produced at diakinesis (Fig. 5d, 1). Bivalents were aligned at the central plate at metaphase I (Fig. 5e) and separated at telophase I (Fig. 5f, o). At metaphase II, sister chromatids were placed at the equatorial plate (Fig. 5g), and the second meiotic division was completed. Finally, four daughter cells were produced at the tetrad stage (Fig. 5h).

Throughout the entire course of meiosis, normal chromosome behaviors were observed in most PMCs of BB\#50. However, it is notable that univalent chromosomes were sporadically detected at both metaphase I and II (Fig. 5m, p), and notably, an interchromosomal bridge was occasionally formed at anaphase I in BB\#4 PMCs (Fig. 5n). Therefore, dysregulation of meiosis such as the formation of univalents and inter-chromosomal bridges may cause chromosome aberrations (Fig. 5q), and these abnormalities in chromosome segregation may induce the formation of unbalanced tetrads or polyads. In BB\#50, a normal meiotic chromosome behavior was observed in the majority of PMCs, whereas $25.0 \%(n=48), 25.0 \%(n=24)$ and $33.3 \%(n=6)$ of PMCs in BB\#4 displayed abnormal chromosome behaviors at metaphase I, anaphase I and metaphase II, respectively (Table 2). This analysis demonstrates that abnormal segregation of 
a

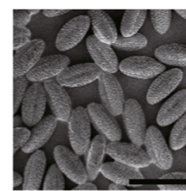

B. rapa

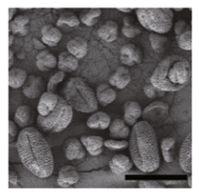

BB\#4

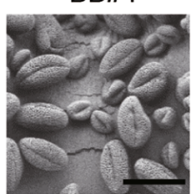

BB\#12

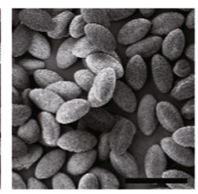

R. sativus

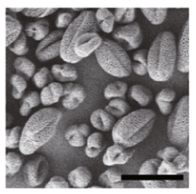

BB\#6

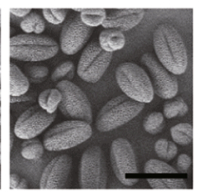

BB\#50 b

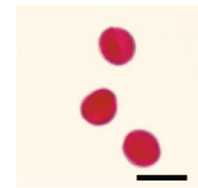

B. rapa

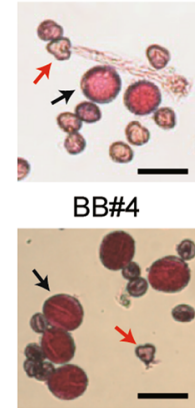

BB\#12

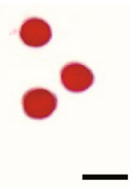

R. sativus

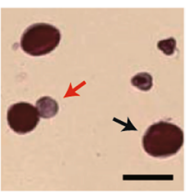

BB\#6

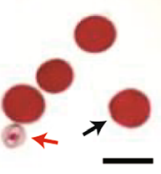

BB\#50

C

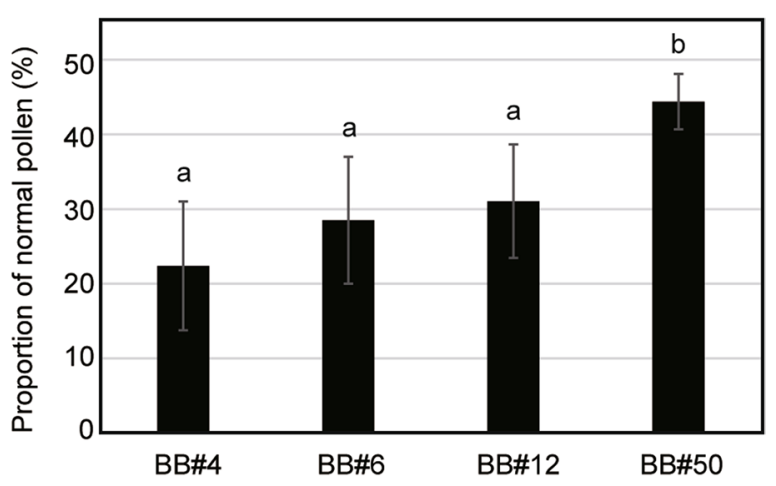

Fig. 3 Variations of pollen viability in xBrassicoraphanus lines. a Shapes of pollen grains in B. rapa, $R$. sativus, BB\#4, BB\#6, BB\#12 and BB\#50. Scale bar $=50 \mu \mathrm{m}$. b Pollen grains stained with Alexander's staining solution. Black and red arrows represent viable and non-viable pollen, respectively. Scale bar $=50 \mu \mathrm{m}$. c Proportion of normal pollen. More than one thousand pollen grains from four independent plants were analyzed for each line. Error bars indicate standard deviations, and differences among xBrassicoraphanus lines were tested with one-way ANOVA, as indicated with letters, by Duncan's multiple test $(p<0.05)$

meiotic chromosomes occurred more frequently in BB\#4 compared to BB\#50 (Fisher's exact test, $p=6.85 \times 10^{-6}$ ) (Table 2). These results also suggest that micronucleus formation observed in BB\#4 is tightly associated with preceding meiotic segregation abnormality, affecting subsequent microspore development.

\section{Identification of the parental origin of univalent chromosomes}

The univalent chromosomes induced by a failure of precise homologous chromosome interactions as observed in BB\#4 (Fig. 5) may produce micronuclei. The parental origins of

chromosomes of BB\#4 were investigated by GISH analysis. PMCs displaying abnormal chromosome behaviors at metaphase I were collected, and chromosomes derived from $B$. rapa and $R$. sativus were stained in red and green, respectively (Fig. 6). The GISH analysis identified that $93.75 \%$ (15/16 PMCs) of univalent chromosomes in BB\#4 had origins of $B$. rapa. This observation suggests that, as in many newly synthesized allopolyploids, a subset of parent-of-origin-specific chromosomes may preferentially participate in non-homologous pairing and cause abnormal segregation during gamete production.

\section{Discussion}

Interspecific hybridization has been utilized in plant breeding to transfer desirable traits between species, producing novel phenotypes with the desire of heterosis. Although several spontaneous interspecific hybrids have occurred in nature as exemplified by the model of 'triangle of U', artificially synthesized hybrid plants frequently exhibit reduced viability and sterility (Bing et al. 1996; García-Fortea et al. 2019; Monteiro et al. 2011; Warwick et al. 2003). The failures of hybrids to produce viable seeds or fertile pollen grains are due in part to the hybridization barrier which is a major hindrance to hybrid breeding. Hybridization barriers are categorized into pre- and post-zygotic barriers according to the timing of fertilization. Pre-zygotic barriers are engaged prior to fertilization between egg and sperm within the embryo sac, mostly preventing pollen germination and/ or pollen tube growth and guidance. Post-zygotic barriers act after fertilization and are usually manifested as poor development of the zygote and sterility of the hybrid offspring.

In this study, we observed several reproduction-related defects which may signify post- zygotic barriers in synthetic $\mathrm{x}$ Brassicoraphanus lines. Few morphological differences were observed among them, but seed development and pollen viability were significantly different between lines $\mathrm{BB} \# 50$ and BB\#4 (Figs. 2, 3), suggesting that post-zygotic barriers were unequally expressed in each offspring. Seed abortion in hybrids is likely caused by a failure in endosperm development or by dysregulation of embryogenesis in the hybrid zygote (Kradolfer et al. 2013; Lafon-Placette et al. 2017; Rebernig et al. 2015).

Importantly, hybrid sterility is often associated with abnormal meiosis during gamete formation (Meena et al. 2017; Mendes-Bonato et al. 2007; Ohmido et al. 2015). It was previously reported that newly synthesized $B$. napus are frequently burdened with massive chromosome rearrangements such as translocation, deletion, insertion and aneuploidy (Gaeta et al. 2007; Xiong et al. 2011). Abnormalities in meiosis associated with poor pollen viability are also reported in a variety of synthetic hybrid plants (Meena 


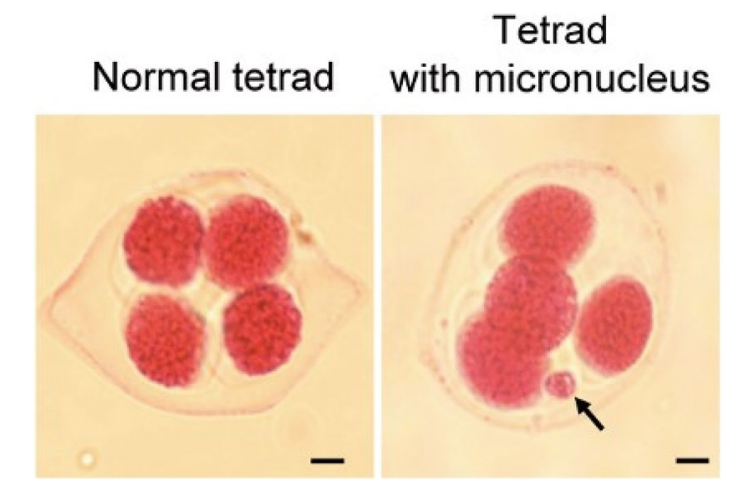

Fig. 4 Tetrad and micronucleus formation in xBrassicoraphanus PMCs. A normal tetrad (left) and a tetrad with a micronucleus (right) are shown. Arrow indicates the micronucleus. Scale bar $=10 \mu \mathrm{m}$

Table 1 Frequency of tetrads with micronucleus

\begin{tabular}{lll}
\hline Lines & Normal tetrad \% & $\begin{array}{l}\text { Tetrad with micronu- } \\
\text { cleus \% }\end{array}$ \\
\hline BB\#4 & $93.02(240 / 258)^{\mathrm{a}}$ & $6.98(18 / 258)^{\mathrm{b}}$ \\
BB\#6 & $95.21(338 / 355)^{\mathrm{a}}$ & $4.79(17 / 355)^{\mathrm{b}}$ \\
BB\#12 & $97.72(472 / 483)^{\mathrm{a}}$ & $2.28(11 / 483)^{\mathrm{b}}$ \\
BB\#50 & $99.26(404 / 407)^{\mathrm{a}}$ & $0.74(3 / 407)^{\mathrm{b}}$ \\
\hline
\end{tabular}

${ }^{a}$ Number of normal tetrads/number of observed PMCs

${ }^{b}$ Number of tetrads with micronucleus/number of observed PMCs et al. 2017; Mendes-Bonato et al. 2007; Ohmido et al. 2015). In our cytological analysis, abnormal chromosome segregation was intermittently observed in PMCs of BB\#4 (Fig. 5; Table 2), with a more frequent formation of micronuclei than in BB\#50 (Fig. 4; Table 1). The micronucleus is a small nuclear mass containing DNA and surrounded by a membrane, which can be generated from an isolated chromosome or chromosome fragments. Therefore, tetrads containing micronuclei may represent unbalanced chromosome complements, consequently imposing detrimental effects on subsequent pollen development. In our study, the formation of micronuclei in $\mathrm{x}$ Brassicoraphanus lines was positively correlated with pollen deformation, suggesting that the persistence of micronuclei induced by a meiotic failure severely compromised pollen viability observed in BB\#4.

The chromosomes forming univalents at metaphase I and II in xBrassicoraphanus PMCs were mostly derived from B. rapa $(93.75 \%)$ (Fig. 6). This observation suggests that specific chromosomes or fragments might trigger nonhomologous interactions, resulting in meiotic abnormality during chromosome segregation. In resynthesized B. napus, aneuploidy was frequently caused by homoeologous interactions between the chromosomes sharing a high level homology in sequence and structure. For instance, syntenies of A1 and $\mathrm{C} 1$, and $\mathrm{A} 2$ and $\mathrm{C} 2$ chromosomes (A from $B$. rapa and $\mathrm{C}$ from B. oleracea) are highly conserved (Parkin et al. 2005), and a deletion or insertion of such chromosomes was highly recurrent in early generations of resynthesized $B$. napus

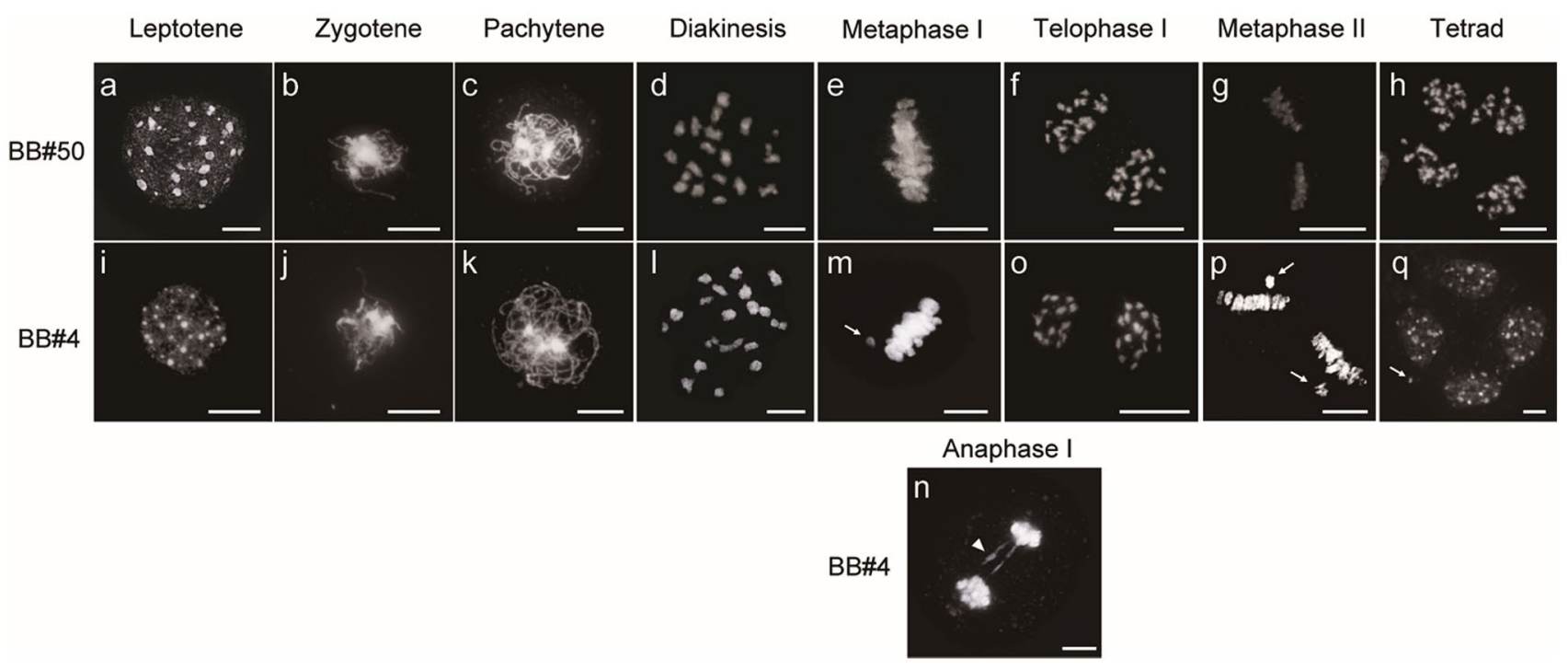

Fig. 5 Meiotic chromosome behaviors in PMCs of BB\#50 and BB\#4. The chromosomes in PMCs of BB\#50 (a-h) and BB\#4 (i-q) were stained with DAPI. Chromosomes were condensed at leptotene (a and i) and thin threads were observed at zygotene (b and $\mathbf{j}$ ). The synapsis was formed at pachytene (c and $\mathbf{k}$ ) and bivalents produced at diakinesis (d and $\mathbf{l}$ ). All chromosomes were located at the central plate at metaphase I (e) and separated at telophase I (f and o). All chromosomes were aligned at the central plate at metaphase II (g). Four microspores were produced at the tetrad stage (h and $\mathbf{q})$. Univalent chromosomes (white arrows) and chromosome bridges (arrowhead) were observed at metaphase I, anaphase I, metaphase II and tetrad stages of BB\#4 (m, n, p and $\mathbf{q})$. Scale bar $=10 \mu \mathrm{m}$ 
Table 2 Frequency of abnormal meiosis in BB\#4 and BB\#50

\begin{tabular}{llllll}
\hline Stage & BB\#4 & & BB\#50 & \\
\cline { 2 - 3 } & Abnormality $\%$ & Normality $\%$ & & Abnormality $\%$ & Normality \% \\
\hline Metaphase I & $25.0(12 / 48)^{\mathrm{a}}$ & $75.0(36 / 48)^{\mathrm{b}}$ & & $1.9(1 / 52)^{\mathrm{a}}$ & $98.1(51 / 52)^{\mathrm{b}}$ \\
Anaphase I & $25.0(6 / 24)^{\mathrm{a}}$ & $75.0(18 / 24)^{\mathrm{b}}$ & & $0(0 / 18)^{\mathrm{a}}$ & $100(18 / 18)^{\mathrm{b}}$ \\
Metaphase II & $33.3(2 / 6)^{\mathrm{a}}$ & $66.7(4 / 6)^{\mathrm{b}}$ & & $0(0 / 4)^{\mathrm{a}}$ & $100(4 / 4)^{\mathrm{b}}$ \\
\hline
\end{tabular}

${ }^{a}$ Number of PMCs showing abnormal meiosis/number of observed PMCs

${ }^{\mathrm{b}}$ Number of PMCs showing normal meiosis/number of observed PMCs
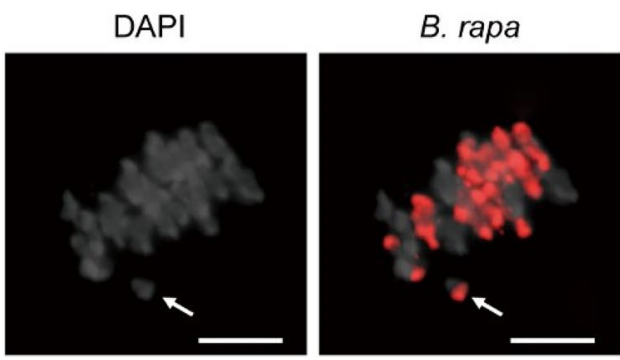

Fig. 6 Univalent formation at metaphase I in the BB\#4 PMC. Genomic DNA isolated from B. rapa and $R$. sativus was labeled with digoxigenein-11-dUTP (red) and biotin-16-dUTP (green) by nick

(Xiong et al. 2011). In the genome of xBrassicoraphanus, albeit an intergeneric hybrid between distantly related species, chromosomes A8 and R8 have syntenic homology (Park et al. 2020). This suggests the possibility that univalent chromosomes in BB\#4 are generated by the persistence of non-homologous interactions, even between parental chromosomes. In the end, stabilization of hybrid genome of $\mathrm{x}$ Brassicoraphanus may require an additional process at the chromatin level, for which epigenetic modifications such as DNA methylation and chromatin remodeling should play an important role to suppress undesirable stand exchanges between non-homologous chromosomes during meiosis.

Acknowledgements This work was supported by the Next-Generation BioGreen 21 Program (PJ013262) and the National Agricultural Genome Program (PJ013440) by Rural Development Administration (RDA).

\section{Compliance with ethical standards}

Conflict of interest The authors declare that they have no conflicts of interests.

Open Access This article is licensed under a Creative Commons Attribution 4.0 International License, which permits use, sharing, adaptation, distribution and reproduction in any medium or format, as long as you give appropriate credit to the original author(s) and the source, provide a link to the Creative Commons licence, and indicate if changes were made. The images or other third party material in this article are included in the article's Creative Commons licence, unless indicated otherwise in a credit line to the material. If material is not included in the article's Creative Commons licence and your intended use is not

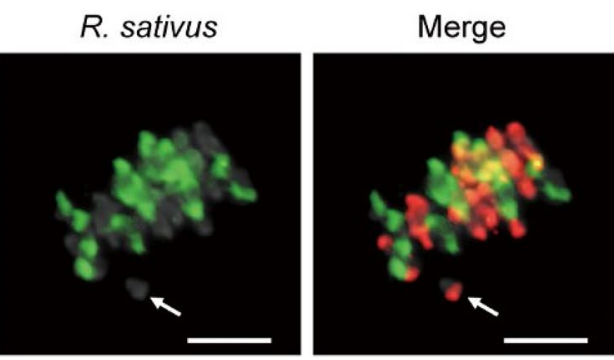

translation, respectively, and used as a probe for GISH analysis. An arrow indicates a univalent chromosome. Scale bar $=10 \mu \mathrm{m}$

permitted by statutory regulation or exceeds the permitted use, you will need to obtain permission directly from the copyright holder. To view a copy of this licence, visit http://creativecommons.org/licenses/by/4.0/.

\section{References}

Abbott R, Albach D, Ansell S, Arntzen JW, Baird SJ, Bierne N, Boughman J, Brelsford A, Buerkle CA, Buggs R et al (2013) Hybridization and speciation. J Evol Biol 26:229-246

Alexander MP (1969) Differential staining of aborted and nonaborted pollen. Stain Technol 44:117-122

Bing D, Downey R, Rakow G (1996) Hybridizations among Brassica napus, $B$. rapa and $B$. juncea and their two weedy relatives $B$. nigra and Sinapis arvensis under open pollination conditions in the field. Plant Breed 115:470-473

Chalhoub B, Denoeud F, Liu S, Parkin IA, Tang H, Wang X, Chiquet J, Belcram H, Tong C, Samans B et al (2014) Early allopolyploid evolution in the post-Neolithic Brassica napus oilseed genome. Science 345:950-953

Chen ZJ (2007) Genetic and epigenetic mechanisms for gene expression and phenotypic variation in plant polyploids. Ann Rev Plant Biol 58:377-406

Chen S, Ren F, Zhang L, Liu Y, Chen X, Li Y, Zhang L, Zhu B, Zeng $\mathrm{P}, \mathrm{Li} \mathrm{Z}$ et al (2018a) Unstable allotetraploid tobacco genome due to frequent homeologous recombination, segmental deletion, and chromosome loss. Mol Plant 11:914-927

Cheng F, Wu J, Cai X, Liang J, Freeling M, Wang X (2018b) Gene retention, fractionation and subgenome differences in polyploid plants. Nat Plants 4:258-268

Cifuentes M, Eber F, Lucas MO, Lode M, Chevre AM, Jenczewski E (2010) Repeated polyploidy drove different levels of crossover suppression between homoeologous chromosomes in Brassica napus allohaploids. Plant Cell 22:2265-2276 
Dion-Cote AM, Barbash DA (2017) Beyond speciation genes: an overview of genome stability in evolution and speciation. Curr Opin Genet Dev 47:17-23

Edger PP, Poorten T, VanBuren R, Hardigan MA, Colle M, McKain MR, Smith RD, Teresi S, Nelson ADL, Wai CM et al (2019) Origin and evolution of the octoploid strawberry genome. Nat Genet 51:541

Gaeta RT, Pires JC, Iniguez-Luy F, Leon E, Osborn TC (2007) Genomic changes in resynthesized Brassica napus and their effect on gene expression and phenotype. Plant Cell 19:3403-3417

García-Fortea E, Gramazio P, Vilanova S, Fita A, Mangino G, Villanueva G, Arrones A, Knapp S, Prohens J, Plazas M (2019) First successful backcrossing towards eggplant (Solanum melongena) of a New World species, the silverleaf nightshade (S. elaeagnifolium), and characterization of interspecific hybrids and backcrosses. Sci Hortic 246:563-573

Goda DIG (2018) Taxonomic importance of pollen morphology for some species of Brassicaceae. Pak J Biol Sci 21:215-223

Hossain M, Inden H, Asahira T (1990) Pollen morphology of interspecific hybrids ofBrassica oleracea and B. campestris. HortScience 25:109-111

International Wheat Genome Sequencing C (2014) A chromosomebased draft sequence of the hexaploid bread wheat (Triticum aestivum) genome. Science 345:1251788

Kradolfer D, Wolff P, Jiang H, Siretskiy A, Kohler C (2013) An imprinted gene underlies postzygotic reproductive isolation in Arabidopsis thaliana. Dev Cell 26:525-535

Lafon-Placette C, Johannessen IM, Hornslien KS, Ali MF, Bjerkan KN, Bramsiepe J, Glöckle BM, Rebernig CA, Brysting AK, Grini PE (2017) Endosperm-based hybridization barriers explain the pattern of gene flow between Arabidopsis lyrata and Arabidopsis arenosa in Central Europe. Proc Natl Acad Sci USA 114:E1027-E1035

Lee S, Choi W, Woo J (2002) Development of a new vegetable crop in $\mathrm{xBrassicoraphanus}$ by hybridization of Brassica campestris and Raphanus sativus. Korean Soc Hort Sci 43:693-698

Lee SS, Lee SA, Yang J, Kim J (2011) Developing stable progenies of $\mathrm{x}$ Brassicoraphanus, an intergeneric allopolyploid between Brassica rapa and Raphanus sativus, through induced mutation using microspore culture. Theor Appl Genet 122:885-891

Lee S-S, Hwang BH, Kim TY, Yang J, Han NR, Kim J, Kim HH, Belandres HR (2017) Developing stable cultivar through microspore mutagenesis in $\times$ Brassicoraphanus koranhort, intergeneric allopolyploid between Brassica rapa and Raphanus sativus. Am J Plant Sci 8:1345-1356

Martinez-Perez E, Colaiacovo MP (2009) Distribution of meiotic recombination events: talking to your neighbors. Curr Opin Genet Dev 19:105-112

Masterson J (1994) Stomatal size in fossil plants: evidence for polyploidy in majority of angiosperms. Science 264:421-424

Meena H, Sujatha M, Soni PK (2017) Interspecific hybrid between cultivated sunflower (Helianthus annuus L.) and silver leaf sunflower H. argophyllus T. \& G.: Cytomorphological and molecular characterization. Indian J Genet 77:547-555

Mendes-Bonato AB, Pagliarini MS, Do Valle CB (2007) Meiotic arrest compromises pollen fertility in an interspecific hybrid between Brachiaria ruziziensis $\mathrm{x}$ Brachiaria decumbens (Poaceae : Paniceae). Braz Arch Biol Technol 50:831-837

Monteiro CES, Pereira TNS, Campos KP (2011) Reproductive characterization of interspecific hybrids among Capsicum species. Crop Breed Appl Biotechnol 11:241-249

Murashige T, Skoog F (1962) A revised medium for rapid growth and bio assays with tobacco tissue cultures. Physiol Plant $15: 473-497$
Ohmido N, Ueda K, Fujii K (2015) Chromosome instability of allopolyploid resynthesized Brassica napus. Chromosome Sci 18:79-84

Park HR, Park JE, Kim JH, Shin H, Yu SH, Son S, Yi G, Lee SS, Kim HH, Huh JH (2020) Meiotic chromosome stability and suppression of crossover between non- homologous chromosomes in $\mathrm{x}$ Brassicoraphanus, an intergeneric allotetraploid derived from a cross between Brassica rapa and Raphanus sativus. Front Plant Sci 11:851

Parkin IA, Gulden SM, Sharpe AG, Lukens L, Trick M, Osborn TC, Lydiate DJ (2005) Segmental structure of the Brassica napus genome based on comparative analysis with Arabidopsis thaliana. Genetics 171:765-781

Rebernig CA, Lafon-Placette C, Hatorangan MR, Slotte T, Kohler C (2015) Non-reciprocal interspecies hybridization barriers in the Capsella genus are established in the endosperm. PLoS Genet 11:e1005295

Soltis PS, Soltis DE (2009) The role of hybridization in plant speciation. Annu Rev Plant Biol 60:561-588

Szadkowski E, Eber F, Huteau V, Lode M, Huneau C, Belcram H, Coriton O, Manzanares- Dauleux MJ, Delourme R, King GJ et al (2010) The first meiosis of resynthesized Brassica napus, a genome blender. New Phytol 186:102-112

Todesco M, Pascual MA, Owens GL, Ostevik KL, Moyers BT, Hubner S, Heredia SM, Hahn MA, Caseys C, Bock DG et al (2016) Hybridization and extinction. Evol Appl 9:892-908

UN (1935) Genome analysis in Brassica with special reference to the experimental formation of $B$. napus and peculiar mode of fertilization. Jpn J Bot 7:389-452

Van de Peer Y, Mizrachi E, Marchal K (2017) The evolutionary significance of polyploidy. Nat Rev Genet 18:411-424

Warwick S, Simard M-J, Légère A, Beckie H, Braun L, Zhu B, Mason P, Séguin-Swartz G, Stewart C (2003) Hybridization between transgenic Brassica napus L. and its wild relatives: Brassica rapa L., Raphanus raphanistrum L., Sinapis arvensis L., and Erucastrum gallicum (Willd.) OE Schulz. Theor Appl Genet 107:528-539

Wendel JF (2000) Genome evolution in polyploids. Plant Mol Biol 42:225-249

Xiong Z, Gaeta RT, Pires JC (2011) Homoeologous shuffling and chromosome compensation maintain genome balance in resynthesized allopolyploid Brassica napus. Proc Natl Acad Sci USA 108:7908-7913

Yang J, Liu D, Wang X, Ji C, Cheng F, Liu B, Hu Z, Chen S, Pental D, $\mathrm{Ju} Y$ et al (2016) The genome sequence of allopolyploid Brassica juncea and analysis of differential homoeolog gene expression influencing selection. Nat Genet 48:1225-1232

Zhang H, Bian Y, Gou X, Zhu B, Xu C, Qi B, Li N, Rustgi S, Zhou H, Han F et al (2013) Persistent whole-chromosome aneuploidy is generally associated with nascent allohexaploid wheat. Proc Natl Acad Sci USA 110:3447-3452

Zhang TZ, Hu Y, Jiang WK, Fang L, Guan XY, Chen JD, Zhang JB, Saski CA, Scheffler BE, Stelly DM et al (2015) Sequencing of allotetraploid cotton (Gossypium hirsutum L. acc. TM-1) provides a resource for fiber improvement. Nat Biotechnol 33:531-U252

Publisher's Note Springer Nature remains neutral with regard to jurisdictional claims in published maps and institutional affiliations. 